\title{
How editors edit
}

\author{
We shed some light on how the Nature Methods editorial team evaluates papers submitted to the journal.
}

T he life of a professional scientific journal editor is exciting, challenging, and intellectually stimulating, but it requires a thick skin. We've been praised by some authors for our behind-the-scenes work, but we've also been called "paper pushers"-and worse. At conferences we are often approached by researchers curious about what it is that we do. We also receive comments such as, "Do you realize just how much power you have?" Editors should be viewed not as obstacles to publication, but as partners with the research community, tasked with curating, improving, and disseminating important, interesting, and high-quality work. Yet as we demand more transparency from our authors, we appreciate that we must also provide more insight into our own editorial processes, and so here we outline how the Nature Methods editorial team evaluates submitted papers.

Nature Methods has no external editorial board; all decisions are made by the team of in-house, full-time, $\mathrm{PhD}$ editors. To keep abreast of current trends and challenges, we travel to multiple conferences each year, visit institutes, and invite researchers to the office. We closely follow community cultures in enforcing standards for reporting and data deposition. While we don't discuss specific papers under consideration, we do share general scientific information with other editors at Nature Research. In short, we spend our days entrenched in the fields that we cover. But because we are not active scientists, we have no competing scientific or financial interests that might bias us toward or against a manuscript.

When a paper is submitted to Nature Methods, the chief editor assigns it to the editor with the most relevant expertise. The editor reads (yes, we read every paper that is submitted, and not just the abstract!), summarizes, and discusses the paper with at least one other editor with related expertise. Together, they make a decision to reject the paper or send it for peer review, and the lead editor communicates that decision to the author (usually within one week of submission).

We look for papers that demonstrate novel approaches to challenging and highinterest problems in basic biology research, enabling researchers to explore new corners of biology, or that solve nagging technical challenges. We exclude work that does not fall within this scope. Besides scope, novelty, potential interest, and practical value, we look at the meat of the paper: have the authors justified the advance over previous methods? Have they appropriately validated the method's performance? Have they done experiments to showcase cool new applications? If a paper is lacking in one of these areas, this does not necessarily spell its end; we may review a promising paper that needs more work, or we might suggest further experiments that could make it a stronger candidate for peer review. However, we send only about $12 \%$ of submitted papers out for peer review. This means that we reject many good-quality papers that just don't quite reach our bar, but we often recommend transfers to other journals in the Nature Research family that might be more suitable.

Many of the papers that we consider are multidisciplinary, so we solicit opinions not only from technical experts, but also from biologists who represent 'end users' of the technology. Authors' requests to exclude reviewers are always honored, as long as the list is reasonable. We are continually exploring new ways to improve peer review, such as our pilot project with Code Ocean to facilitate the review of code. We allow reviewers to reveal their identities to authors, should they so choose, and we also offer double-blind peer review as an author choice.

When a paper comes back from peer review, the editor summarizes the reviewers' comments and discusses her or his recommendation for revision, rejection with potential for resubmission, or complete rejection with at least one other team member. The chief editor is also involved in any recommendations for revision. Our decisions are never based on reviewer 'votes'. We read the reviewers' comments in light of our initial assessment of interest, novelty, validation, and application. We evaluate which concerns are crucial to address and which are not, and communicate this to the authors. Occasionally, we ask reviewers for further input on technical issues, or we may ask authors for a revision plan prior to making a decision. We strive to be open; if our expectations for a revision are unclear, we welcome further discussion by e-mail or phone.
We realize that reviewers can occasionally make mistakes or show signs of bias, and authors can address serious technical concerns that prompted us to reject an otherwise interesting paper. Therefore, we do consider appeals of rejected papers, though authors should very carefully consider the reasons the editor has stated for rejection and whether they can adequately address these concerns.

In the best-case scenario, the process from submission to acceptance will take just a few months. Occasionally papers go through multiple revisions over the course of a year or more, though we try to avoid more than two rounds of peer review. About half of the papers initially sent for peer review are published in Nature Methods. In the final stage just prior to acceptance, the editor carefully reads the paper and suggests wording changes to help clarify and focus the message or tone down claims that are not supported by the data, as well as ensures that our standards for reporting and for software, data, and materials availability are enforced.

To help recognize the dedication that the team puts into editing each paper, and to promote further transparency in our editorial processes, we will soon begin adding the editors' names to papers that we publish in Nature Methods.

We also do more than edit papers. We highlight interesting research published both in our own pages and in other journals with Research Highlights, News \& Views, and Technology Features, as well as via social media. We aim to educate about and raise awareness of scientific and publishing issues through our Editorials and columns such as the Points of Significance. We commission Reviews and Perspectives of broad utility for scientists.

At the heart of our profession is an obligation to provide a useful service to the research community; we welcome feedback about how we can further improve. We take our roles with great responsibility and are proud to support and promote exciting and high-quality research.

Published online: 30 January 2019 https://doi.org/10.1038/s41592-019-0324-z 